\title{
O Ensino de Práticas Não-Convencionais em Saúde nas Faculdades de Medicina: Panorama Mundial e Perspectivas Brasileiras
}

\author{
The Teaching of Non-Conventional \\ Practices regarding Health Care in Medical \\ Schools: World Scenario and Brazilian \\ Perspectives
}

Marcus Zulian Teixeira

Chin An Lin $^{2}$

Milton de Arruda Martins ${ }^{3}$

WRASCDIAVIE

Educaţào Miedica;

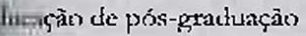

tinedicins:

intaina complenentar;

boneoparix;

Ampuniam:

immite;

inriculo.

WoWRDS:

ucalion, Medical;

Fllucation, medical,

thlignduate;

implementary therapies;

thmeopathy;

houpuncture;

Atntude;

aifricitham

ifr) $(\pi): 23 / 06 / 2003$

mirihado cm: 25/09/2003

Jlo cerI 14/1? ?/2003

${ }^{2}$ Médico pesquisador, Deprutamento de Clinica Médica, Hospital das Clinicas te Faculdarte de Medicinu da Lintreersidode de Säu Paulo, Săo Paulo. Brasil.

"Médico assistente, projessor colaborudor, Departamento de Clinica Médica, Hospital das Clifisicas da Faculdade de Medicina da Universidede de Sao Paulo, Š̆o Paulo, Brasil.

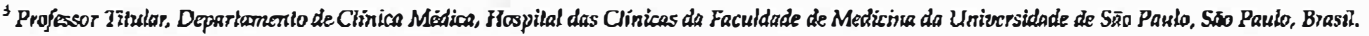

\begin{abstract}
The world population's demand for non-corventional practices regarding health care augmented subs. tantially over the last decade, the doctors are being increasingly required to have the basic notions concerning the current diversified therapeutics, so that they can guide the patients under their care that want to use treatments which are different from those habitually used by them. In this case the medical schuols have to give the undergraduate and postgraduate students the knowtedge referring to the scientific evidances, the theoretical presuppositions and the clinical and therapeutic approaches used in these different treatment forms. The present seview has the pupose of fomenting the discussion segarding the teaching of non-conventional medical practices (homeopathy and acupuncture) in Brazilian Mcdical Schools. It is focused on the interest of the population and of the medical class (students, residents and specialists) in these referred therapeutics, on the importance of Brazilian initiatives, as well as those of other countries concerning undergraduate, postgraduate and residence teaching, and on the perspectives regarding medical education in terms of non-conventional practices in health care.
\end{abstract}




\section{INTRODUÇĀO}

Nos Estados Unidos, o National Center for Complementary and Alternative Medicine, do National Institutes of Health $(\mathrm{NIH})$, define "complementary and alternative medicines" (CAM) ${ }^{1}$ como "aqueles tratamentos e práticas de atenção à saúde que não são amplamente ensinados nas escolas médicas, não săo geralmente utilizados em hospitais e não são usualmente reembolsados pelas empresas de seguro médico", englobando uma diversidade de práticas incomuns de cuidados com a saúde (homeopatia; acupuntura; fitoterapia; quiroprática; hipnose; biofeedback; meditação e relaxamento; espiritualismo, religião e oração; toque terapêutico; nutriçāo e dietas; medicina naturałjsta; osteopatia; massagem terapêutica e outras).

Tanto a classe médica quanto a população brasileira costumam englobar as distintas formas de tratamento médico não-convencional segundo a denominação de "medicina alter nativa", termo adotado no passado pelos EUA e que não corresponde à realidade prática de algumas destas terapêuticas.

$\mathrm{O}$ adjetivo "alternativa" significa "o que se diz ou faz com alternação" ou "que vem ora um, ora outro" ou "que não está ligado aos interesses ou tendências políticas dominantes", descartando, de acordo com a primeira definição, a atuação conjunta entre as diversas técnicas terapêuticas não-tradicionais e os procedimentos médicos convencionais, contrariando o pensamento e a conduta dos propagadores destas práticas ${ }^{3}$. Estes defendem uma atuação conjunta e integra tiva (coadjuvante, não-excludente) entre todas as formas de tratamento possiveis e confiáveis, buscando proporcionar ao paciente uma cura mais suave, profunda e duradoura. Se pensarmos no último significado, que se refere a práticas médicas não-usuais, não-convencionais, não-tradicionais, "não-dominantes", o termo poderia ser utilizado.

A denominaçāo "medicina complementar", adotada no Reino Unido e, atualmente, nos EUA (CAM), também não condiz com a atuação destas práticas no tratamento das enfermidades humanas, pois o adjetivo "complementar" significa "que serve de complemento" ou "que sucede ao elementar", em contradiçăo às evidências clínicas que apontam para a resolutividade de diversos problemas de saúde tratados exclusivamente por práticas médicas não-convencionais.

$\mathrm{Na}$ implantaçāo do "Grupo de Estudos e Pesquisas em Práticas Não-Convencionais em Saúde", no Serviço de Clínica Geral do Hospital das Clínicas da Faculdade de Medicina da Unjversidade de Sāo Paulo, resolvemos adotar a denominação "práticas não-convencionais em saúde" (PNCS) para estas atitudes terapêuticas distintas das "amplamente ensinadas nas faculdades de medicina e geralmente utilizadas em hospitais". Isto porque, com o avanço das pesquisas cientifl cas, elas poderāo vir a ocupar posições de destaque frente av demais tratamentos médicos, passando a ser consideradn "convencionais".

No Brasil, tanto a homeopatia como a acupuntura si consideradas especialidades médicas reconhecidas pelo $C$ con selho Federal de Medicina (CFM) desde 1980 e 1995, respecti vamente, com a consulta e os procedimentos médicos (reper torização na homeopatia e aplicação das agulhas na acupun tura) "reembolsados pelas empresas de seguro médico", ser: do oferecidas ambulatorialmente na rede pública de saúch (postos de saúde e alguns hospitais). Segundo levantament brasileiro, realizado pelo convênio Fíocruz/CFM (1996) 4 . homeopatia, como especialidade principal de atuação, ocupz o $16^{\circ}$ maior contingente de profissionais dentre 61 especial dades analisadas.

Com o crescimento de iniciativas, no ensino e na pesqu sa universitária, que visam aproximar a homeopatia e a acı puntura do ambiente acadêmico, compete à dedicaçāo du propagadores destas práticas e à vontade política dos condí tores do ensino médico o fato de virem a se tornar "convend onais", a partir de sua inclusão no currículo da graduaç̧ิ̄o da pós-graduação das faculdades de medicina, podendo v'ir figurar, futuramente, como programa de residência médiç

Esta revisão tem o propósito de fomentar a discussảo 50 bre o ensino de práticas médicas não-convencionais (homed patia e acupuntura) nas faculdades de medicina brasileira evidenciando o interesse da população e da classe médica fe tudantes, residentes e especialistas) nestas terapéuticas, a in portância e as iniciativas brasileiras e de outros países no sino na graduação, na pós graduação e na residência, e perspectivas para o futuro da educação médica em prátic não-convencionais em saúde.

\section{INTERESSE E IMPORTÂNCIA DO ENSINO DE PNCS NA GRADUAÇÃO MÉDICA}

Interesse e Emprego das PNCS pela População Mundial

Em 1990, Eisenberg e colaboradores realizaram o prime ro levantamento ${ }^{5}$ sobre a prevalência, os custos e as forma de utilização de PNCS nos EUA, estimando que $34 \%$ da po pulação adulta americana as empregavam, num total de $t^{2}$ milhōes de consultas/ano com terapeutas de PNCS.

Repetiram a pesquisa em 1997, estimando um aumen! da utilização das PNCS ( $42 \%$ da população), com 629 milhổ de consultas a terapeutas (excedendo o número total de con sultas do sistema primário de saúde dos EUA). Isto represer tava um custo adicional de US $\$ 27$ bilhōes à população amen cana, por estas práticas não estarem disponíveis nos serviłł 
públicos de saúde e não serem reembolsadas pelas empresas de seguro médico ${ }^{\circ}$.

Una pesquisa realizada na Europa ${ }^{7}$ mostrou que $\$ 6 \%$ da população da Alemanha faziam uso de PNCS, enquanto na França o índice era de $49 \%$.

Num levantamento por telefone, que buscou avaliar o uso de 11 PNCS por moradores da Florida ${ }^{8}$ (EUA), a taxa de resposta foi $54 \%$, dos quais $62 \%$ afirmaram ter utilizado uma ou mais destas terapias. Em estudo prévio do mesmo autor', a maior incidência de uso recaiu sobre a população feminina, solteira, com a saúde afetada e freqüentando médicos convencionais regularmente. Relacionando as PNCS mais empregadas, $31 \%$ tinham utilizado remédios caseiros, $24 \%$ dietas especiais, $20 \%$ técnicas de relaxamento e $18 \%$ medicinas herbárias. Acupuntura, biofeedback, medicina energética e hipnose foram usadas por menos de $5 \%$ da amostra.

Em levantamentos ${ }^{10.11}$ que indagaram os motivos que levavam a população americana a procurar as terapias não-usudis, a insatisfação dos pacientes com a medicina convencional éapontada como a principal justificativa para o aumento progressivo do interesse pelas PNCS. Encontrou-se também que as pessoas buscavam uma orientação filos6fica holística a respeito do binômio saúde-doença, que explicasse as doenças através da conexão corpo-mente-espírito ${ }^{12}$.

Em 1998, o Congresso dos EUA autorizou a transformação do Office of Alternative Medicine do National Institutes of Health (NIH), após seis anos de funcionamento, no National Center for Complementary and Alternative Medicine (NC(AM), promovendo um crescimento exponencial das PNCS no pais e disponibilizando um orçamento de US\$ 50 milhões para o desenvolvimento de pesquisa nestas áreas (para o ano de 2003 esta cifra foi de US $\$ 100$ milhōes). A Faculdade de Medicina de Harvard anunciou que vai investir US\$10 milhōes em pesquisas com PNCS.

No Brasil, alguns estudos ${ }^{13,14}$ mostraram que, os pacientes procuram a homeopatia pelos seguintes motivos: insatisfação com a medicina convencional; evitar os efeitos colaterais das drogas clássicas; busca de uma melhora na relação médico-paciente; tratamento que englobe a pessoa como um todo (corpo-mente-espírito).

\section{Importância do Ensino de PNCS para a Classe Médica}

No segundo levantamento ${ }^{6}$ com a população americana, Eisenberg e colaboradores constataram que a maioria dos pacientes que utilizavam PNCS ( $>60 \%$ ) não relatava este fato aos seus médicos.

Pesquisa realizada com pacientes com diagnóstico precoce de câncer de mama ${ }^{15}$ evidenciou que elas evitavam discu- tir o uso concomitante de PNCS com seus médicos, antecipando uma resposta reprovadora dos mesmos, secundária ao desinteresse, ao descrédito e à falta de conhecimento no assunto. As entrevistadas mostraram admiração pelos médicos que respeitam a tomada de decisão da paciente, estão dispostos a escutá-las e procuram manter-se isentos de preconceitos (open-minded). Os autores concluem que o conhecimento das PNCS pelos médicos beneficiaria a relação médico-paciente.

A maioria da classe médica não está apta a responder perguntas ou orientar seus pacientes quanto ao uso de PNCS no que tange aos mecanismos de ação, indicaçōes terapêuticas, interaçōes medicamentosas e efeitos adversos destas terapêuticas ${ }^{16}$.

Fatores adicionais - como o diálogo pobre e inadequado entre médicos convencionais e médicos de PNCS, dúvidas sobre a competência de tais profissionais (pela falta de qualificaçōes prontamente identificáveis e reconheciveis) e o risco de oferecimento de esperança irreal de cura - colocam o paciente numa posição incerta frente às terapêuticas nåo-convencionais. A incorporação sistemática de informaçōes sobre PNCS nos currículos das escolas médicas, com as evidências científicas que respaldam tais tratamentos, além de diminuir o preconceito existente, proveria os médicos futuros com as ferramentas e o conhecimento necessários para permitir que seus pacientes possam se beneficiar adequadamente das PNCS, com riscos limitados ${ }^{17-22}$

Por outro lado, a exploração de tópicos de PNCS no currículo médico ajudaria a elucidar a natureza ampla, complexa e incerta da prática médica, desenvolvendo habilidades nas tomadas de decisōes clínicas, aumentando a sensibilidade cultural e provendo idéias para pesquisas futuras ${ }^{23.24}$.

O emprego de PNCS em doenças crônicas e em outras áreas em que a medicina convencional apresenta, comprovadamente, sucesso limitado significa importante incremento ao arsenal terapêutico da Medicina ${ }^{25,26}$.

Em iniciativas desenvolvidas na frndia, a utilização de forma integrada das práticas médicas convencionais comas nãoconvencionais evidenciou aumento na qualidade do atendimento e melhora na relação custo/efetividade ${ }^{27}$.

\section{ATITUDES DE MÉDICOS E ACADÊMICOS DE MEDICINA FRENTE ÀS PNCS}

\section{Posicionamento dos Médicos}

Com o aumento da procura da população por PNCS, os médicos estão começando a sentir a necessidade de suprir esta demanda, desviada nos EUA para os terapeutas práticos. O desconhecimento dos fundamentos gerais destas terapêuticas pelos médicos causa frustração nos pacientes que optar 
ram pela utilização de PNCS concomitante ao tratamento convencional, privando-os de uma orientação segura sobre as principais indicaçōes e os riscos (efeitos adversos, interaçōes medicamentosas, etc. $)^{28-30}$ do tratamento que optaram por seguir. O velho aforismo "se não tizer bem, mal não faz", remanescente do movimento naturalista de contracultura, não encontra respaldo no pensamento científico moderno.

A insatisfação dos médicos com o sistema de saúde atual pode ser responsável pelo aumento de seu interesse pelas PNCS, diretamente relacionada ao desgosto dos pacientes pelo mesmo modelo, com aumento progressivo da busca de PNCS pela população mundial ${ }^{3132}$.

Uma metanálise de 12 levantamentos das atitudes de médicos convencionais frente às PNCS mostrou que eles acreditam que as PNCS sāo moderadamente efetivas ${ }^{33}$.

Buscando descrever as atitudes de médicos generalistas perante o uso de PNCS, foi enviado um questionário postal, auto-aplicável, para 800 médicos generalistas de Victoria (Austrália), obtendo-se uma taxa de resposta de $64 \%$ (488). Acupuntura, hipnose e meditação são bem aceitas pelos médicos, tendo sido citadas por mais de $80 \%$ dos seus pacientes, dos quais $50 \%$ as utilizavam. Os médicos referiam treinamento em várias práticas: meditação $(34 \%)$, acupuntura $(23 \%)$, vitamina e terapia mineral (23\%), hipnose (20\%), medicina herbária (12\%), quiroprática ( $8 \%$ ), naturopatia $(6 \%)$, homeopatia $(5 \%)$, cura espiritual (5\%), osteopatia (4\%), aromaterapia (4\%) e reflexologia $(2 \%)$. Em torno de $30 \%$ dos entrevistados manifestaram interesse em aprender quiroprática, medicina herbária, naturopatia e terapia vitamínica-mineral ${ }^{34}$.

De forma análoga, um questionário sobre a experiência pessoal e comunicação com pacientes frente às PNCS foi entregue a 705 médicos de Denver (Colorado, EUA), sendo respondidos e devolvidos por $43 \%$ (302) da amostra: $76 \%$ dos médicos informaram que tinham pacientes que usavam PNCS, $59 \%$ eram questionados sobre PNCS específicas, $48 \%$ tinham recomendado PNCS a pelo menos um paciente e $24 \%$ tinham usado PNCS pessoalmente. A recomendação de PNCS estava fortemente associada à utilizaçāo da terapêutica em si próprios. Poucos médicos sentiam-se confortáveis em discutir PNCS com seus pacientes, e a maioria ( $84 \%$ ) acreditava que precisaria aprender mais sobre PNCS, para enfocar as preocupações dos pacientes adequadamente ${ }^{35}$.

A fim de avaliar as atitudes, $o$ conhecimento e o interesse de pediatras americanos perante as PNCS, foi enviado um questionário de 25 perguntas, auto-aplicável, a 860 pediatras de Michigan (EUA), pertencentes a American Academy of Pediatrics, obtendo-se uma taxa de resposta de 40,5\% (348). Da amostra avaliada, $83,5 \%$ acreditavam que seus pacientes faziam uso de PNCS, sendo que $55,1 \%$ estimavam que isto correspondia a menos de $10 \%$ dos pacientes. Dos que discorreram sobre PNCS com seus pacientes $\{53,8 \%$ do total $), 84,7 \%$ disseram que a discussão, geralmente, foi iniciada pela familia do paciente. Mais da metade dos médicos $(55,2 \%)$ utilizaria PNCS pessoalmente e $50,3 \%$ indicariam $\mathrm{PNCS}$ a seus pacientes. As terapias referidas foram biofeedback $(23,6 \%)$, grapos de auto-ajuda $(23,3 \%)$, relaxamento $(14,9 \%)$, hipnose $(13,8 \%)$ e acupuntura ou acupressura $(10,9 \%)$. Dos médicos que responderam, 54,1\% estavam interessados em freqüentar cursos de educação médica em PNCS. Os autores concluem que, de acordo com as respostas, a formação dos pediatras em PNCS deve ser pensada para o futuro ${ }^{36}$.

\section{Posicionamento dos Acadêmicos de Medicina e Médicos Residentes}

Nưna pesquisa realizada com 592 acadêmicos de medicina da Universidade de Glasgow (Reino Unido) ${ }^{37}, 77 \%$ dos entrevistados consideraram que as PNCS eram úteis e $69 \%$ se mostraram favoráveis à inclusão das mesmas no currículo fundamental.

Um levantamento feito com 204 estudantes de medicina da Universidade de Marburg (Alemanha) evidenciou alto interesse dos entrevistados (80\%) no aprendizado de PNCS, que pretendiam utilizá-las na sua futura prática médica (homeopatia: $45 \%$; acupuntura: $42 \%$; fitoterapia: $33 \%)^{38}$.

Outras pesquisas, realizadas junto a acadêmicos de medicina de diversos paises, também mostraram elevado interesse dos entrevistados no aprendizado de PNCS durante a graduação ${ }^{39-45}$.

Buscando avaliar o programa de quatro anos da residência em medicina familiar integrativa da University of Washington, que associa PNCS ao currículo convencional, foi realizada uma pesquisa junto a 39 médicos residentes do $3^{9}$ e $4^{2}$ anos perguntando sobre a experiência prática, o conhecimento e as atitudes frente às PNCS. Oitenta por cento dos entrevistados achava que a residência deveria prover treinamento em PNCS, e a maioria já havia recomendado alguma PNCS aos seus pa. cientes no último ano ${ }^{46}$.

A Association of American Medical Colleges declarou que os estudantes de medicina devem ter conhecimento suficiente sobre PNCS, para que, ao exercerem a prática médica, estejam aptos a aconselhar seus pacientes a respeito dos possíveis benefícios e malefícios de cada terapeutica ${ }^{47}$.

Numa enquete com 51 estudantes de medicina brasileiros presentes no XXXV Congresso Brasileiro de Educação Médica (1997), Dantas e Ribeiro ${ }^{48}$ buscaram identificar as atitudes de estudantes interessados em ensino médico em rela- 
çảo a quatro práticas não-convencionais utilizadas no Brasil (homeopatia, acupuntura, fitoterapia e hipnose). Mais da metade dos estudantes manifestou aIto interesse em conhecêlas, considerando que deveriam ser ensinadas nas escolas médicas brasileiras de formı opcional (86\%) ou obrigatória $19 \%$ ). Embora mais de $80 \%$ dos entrevistados admitisse estar pouco informado sobre PNCS, $60 \%$ entendiam que tais práticas eram bastante ou extremamente eficazes. Para dois terços da amostra, a homeopatia, a fitoterapia e a acupuntura deveriam estar disponíveis na rede püblica de saúde, cabendo aos médicos a responsabilidade pela indicaçăo e pela prescrição.

Buscando um posicionamento dos acadêmicos de medicina brasileiros frente às PNCS reconhecidas como especialidades médicas em nosso meio (homeopatia e acupuntura), realizamos um levantamento, em 2002, junto aos alunos dos diversos anos da Faculdade de Medicina da Universidade de São Paulo(FMUSP). Um questionário auto-aplicável foi respondido por 484 estudantes, com perguntas sobre interesse no aprendizado e na forma de ensino, nivel de conhecimento e forma de aquisição, experiência e eficácia da terapêutica em si próprios ou em pessoas próximas, principais indicaçōes e eficácia geral, além do oferecimento e integração nos serviços públicos de saúde. Na média das duas disciplinas, acima de $85 \%$ dos estudantes considerou que elas deveriam estar inse. ridas no currículo da graduação das escolas médicas de foruna opcional $(72 \%)$ ou obrigatória (19\%), com $56 \%$ dos entrevistados mostrando-se bastante interessados no aprendizado.

Apesar de nenhum ou pouco conhecimento no assunto (76\%), 67\% acreditavam em algum grau de eficácia, tendo como principais indicaçőes as doenças crônicas, isoladamente (37\%) ou englobando tombém as doenças agudas (29\%). Ao tedor de $35 \%$ dos acadêmicos foram favoráveis ao oferecimento ambulatorial de ambas as especialidades nos serviços públicos de saúde, enquanto a média de $34 \%$ deferdia a oferta destes tratamentos também em hospitais, com $60 \%$ acreditando na possibilidade de integração com a medicina convencional ${ }^{49}$.

\section{ESTIMATTVAS MUNDIAIS DO ENSINO MÉDICO EM PNCS}

Com o aumento da popularidade das PNCS, vem ocorrendo interesse crescente por este tema entre médicos, residentes e estudantes de medicina, os quais sentem necessida. de de recebex instrução nestes assuntos. Escolas médicas e programas de pós-graduação e residência médica estão começando a responder a esta demanda, incluindo o ensino de PNCS em seus currículos, após terem percebido que com esta atitude aumentam a amplitude de ação da medicina e melhoram a relação médico-paciente ${ }^{50}$.
No Reino Unido, a homeopatia dispõe de um estatuto legal que permite a formação pós-graduada de médicos. Em 1993, a British Medical Association ${ }^{51}$ passou a recomendar que as escolas médicas oferecessem cursos de introdução em PNCS para todos os acadêmicos. Três anos após $(1996)^{52}, 23 \%$ das faculdades de medicina do Reino Unido haviam incorporado ao seu curriculo disciplinas que ministravam conceitos básicos sobre PNCS e, em 1999, 40\% das escolas médicas da União Européia ofereciam cursos de $\mathrm{PNCS}^{53}$.

Em 1997, a Ordem dos Médicos da França reconheceu que a homeopatia deveria ser prescrita por médicos que receberam formação no nivel da pós-graduação universitária.

Em alguns estados americanos (Arizona, Nevada e Connecticut), existem autoridades de certificação para homeopatas. O American Institute of Homeopathy confere diploma de homeoterapeutas para médicos, e o Council of Fomeopathic Certification oferece certificação para a homeopatia clássica. A licença para médicos acupunturistas é oferecida em alguns estados americanos.

Acompanhando as mudanças exigidas pela população americana moderna, a última versão do Código de Ética Médica do American College of Physicians incluiu uma seção específica sobre "tratamentos alternativos", recomendando que o médico deve acompanhar o paciente se este decide fazer um tratamento não-convencional ${ }^{54}$.

Nos EUA, é grande o número de escolas médicas que oferecem aulas isoladas em medicina holistica ou $\mathrm{PNCS}^{55}$.

Levantamento realizado em 1995 pela Society of Teachers of Family Medicine junto a 97 escolas médicas dos EUA mos trou que $39,2 \%$ oferecjam alguma forma de instrução em PNCS aos seus residentes, em sua maioria na forma de disciplinas eletivas $(72,2 \%)$. Nas residências não-universitárias para médicos de familia, 28,1\% ofereciam instrução em $\mathrm{PNCS}^{56}$.

No período de 1997-98, uma pesquisa em 117 escolas médicas americanas ${ }^{57}$ mostrou que $64 \%$ ministravam aulas sobre PNCS. Em 1998, levantamento realizado junto às escolas médicas do Canada ${ }^{58}$ mostrou que $81 \%$ delas haviam incorporado tópicos de PNCS ao seu currículo, estando a acupuntura e a homeopatia como as mais freqüentes.

No período 1998-99, uma pesquisa junto a 80 escolas médicas japonesas ${ }^{59}$ mostrou que $20 \%$ (16) ensinavam PNCS, prevalecendo a acupuntura.

Em levantamento recente nos EUA, foram enviados questionários para 123 diretores de cursos em PNCS de 74 escolas médicas, tendo-se obtido resposta junto aos responsáveis de 73 cursos de 53 escolas. Os tópicos mais frequientemente ensinados são acupuntura (76,7\%), fitoterapia (69,9\%), meditação e relaxamento $(65,8 \%)$, espiritualismo/religião/oração $(64,4 \%)$, 
quiroprática (60,3\%), homeopatia (57,5\%) e nutrição/dietas $(50,7 \%)$. A pesar de algurs recebenem mais tempo de instrução, a carga horária média é de duas horas-aula por tópico. $O$ curso de PNCS "típico" está sob a responsabilidade dos Departamentos de Clínica Médica (64,9\%), sendo oferecido na forma de disciplina eletiva (75,3\%), do primeiro ao quarto ano da graduação médica, contemplando menos do que 20 horas-aula de instrução $(52,1 \%)$. O ensino da maioria dos cursos $(78,1 \%)$ é feito por diversos indivíduos identificados como práticos ou prescritores de PNCS. A grande maioria dos cursos tem como objetivo principal ensinar conceitos gerais das PNCS $(61,6 \%)$, e poucos enfatizam as evidências científicas numa avaliação crítica da efetividade das mesmas $(17,8 \%)$ ou oferecem treinamento prático no uso de práticas específicas $(17,8 \%)^{60}$.

No Brasil, apesar de a homeopatia e a acupuntura serem reconhecidas como especialidades médicas, situação privilegiada perante o cenário mundial, em poucas faculdades de medicina brasileiras elas fazem parte do currículo acadêmico, sendo oferecidas, principalmente, na forma de disciplinas optativas.

\section{PROPOSTAS DE ENSINO MEDICO EM PNCS}

\section{Graduação Médica}

$\mathrm{NaAlemanha,} \mathrm{existe} \mathrm{um} \mathrm{projeto} \mathrm{de} \mathrm{integraçāo} \mathrm{da} \mathrm{naturo-}$ patia na pesquisa e no ensino médico universitários. $O$ curso opcional para os estudantes de medicina inclui aulas sobre os fundamentos básicos e as pesquisas desenvolvidas em acupuntura, terapia manual, nutrição, homeopatia, hidroterapia e fitoterapia, abordando as evidências científicas que discorrem sobre os efeitos e a eficácia, indicaçỏes e contra-indicaçōes de cada terapêutica, além de algum treinamento prático em algumas técnicas ${ }^{61}$.

Em Taiwan ${ }^{62}$ e no Japāo ${ }^{63}$, existem propostas para integrar a medicina ocidental à medicina oriental (medicina chinesa) a partir do ensino médico fundamental, acreditando-se que um sistema de cuidados médicos unificado diminuiria os custos gerais com a saúde.

A Universidade do Arizona ${ }^{64}$, pioneira na educação médica em PNCS nos EUA desde 1983, vem oferecendo aos residentes e estudantes do quarto ano da graduação, a partir de 1997, uma disciplina eletiva em "medicina integrativa" com quatro semanas de duração, aprofundando os temas abordados de forma superficial nos primeiros anos do curso e propiciando uma vivência clínica. Propostas semelhantes vêm ocorrendo em outras escolas médicas americanas ${ }^{65,66}$

Segundo o National Center for Complementary and Alternative Medicine (NIH), o ensino de PNCS para acadêmicos de medicina deve estar pautado nos fundamentos básicos de cada modalidade, enfatizando a necessidade do embasa. mento científico para as diversas condutas terapêuticas ${ }^{67}$.

Um consórcio constituído por 11 escolas médicas norteamericanas, com pragramas ativos no ensino de PNCS e medicina integrativa, vem reunindo esforços para incorporar a medicina integrativa ao currículo das escolas e das residéncias médicas. O Consortium of Academic Health Centers for Integrative Medicine (CAHCIM), composto pelas escolas médicas Albert Einstein, Duke, Georgetown, Harvard, Jefferson, Stanford, Arizona, California at San Francisco, Massachusetts, Maryland e Minnesota, tem influenciado o National Board of Medical Examiners para incluir nas suas provas de avaliação questōes sobre PNCS e medicina integrativa.

Diferentes niveis de competência em PNCS são reconhecidos nos EUA: a) baixo, em que os médicos possuem conhecimentos básicos para indicar e encaminhar seus pacientes a outros profissionais mais qualificados; b) médio, em que a habilidade prática permite que os médicos atuem em condiçōes e pałologias específicas; c) alto, em que a competência lhes dá a habilidade para tratar as mais diversas doenças.

No Brasil, a Associaçāo Médica Homeopática Brasileira (AMHB) elaborou um projeto para a implantação de disciplinas eletivas nas escolas de medicina ${ }^{68}$, sugerindo as diretrizes básicas e a grade curricular para um curso com carga horária rúnima de 45 horas-aula.

Citando algumas iniciativas na área, a homeopatia é oferecida como disciplina obrigatória ou optativa nos cursos de medicina da Universidade do Rio de Janeiro (1 obrigatória e 3 optativas, num total de 60 horas-aula); Universidade Federal de Uberlândia (optativa, 40 horas-aula); Universidade Fede ral da Paraíba (optativa, 45 horas-aula); Universidade Federal Fluminense ( 3 optativas, total de 90 horas-aula); Universjdade Federal de Santa Catarina (optativa, 4 hotas-aula); Universidade Federal de São Paulo (optativa, 12 horas aula) Universidade de Săo Paulo (optativa, 60 horas-aula); Universidade do Estodo do Amazonas (optativa, 60 horas-aula) Universidade Federal do Rio Grande do Norte (optativa, 60 horas-aula); Escola de Ciências Médicas de Alagoas (optativa, 60 horas-aula) e Faculdade Evangélica do Paraná (optativa, 40 horas-aula).

A acupuntura tem sido ensinada como disciplina optativa na Faculdade de Medicina de São José do Rio Preto (optativa, 40 horas-aula), na Faculdade Evangélica do Paraná (optativa, 40 horas-aula) e nos cursos de medicina da Universidade Federal de Sāo Paulo (optativa, 12 horas-aula), Universidade de São Paulo (optativa, 60 horas-aula), Universidade Federal de Pernambuco (optativa, 45 horas-aula), Universidade Federal do Piauí (optativa, 40 horas-aula), Universida- 
de Federal Fluminense (optativa, 45 horas-aula) e Universidade Federal do Amazonas (optativa, 45 horas-aula).

\section{Pós-Graduação / Residência Médica}

Nos EUA, a Society of Teachers of Family Medicine Group on Alternative Medicine desenvolveu um consenso de recomendaçōes sobre atitudes, conhecimentos e habilidades em PNCS para serem incorporadas ao treinamento da residência dos médicos de família: 1) influência cultural nas conviç̧ões e nas escolhas sobre saúde; 2) bases teóricas e filosóficas das PNCS; 3) indicações e potenciais efeitos adversos de cada tratamento; 4) evidências científicas de eficácia e custo-benefício de cada modalidade ${ }^{69}$.

Em 1996, surgiu na University of Arizona College of Medicine uma nova proposta para a educaçăo médica, intitulada "medicina integrativa", definida como uma "medicina que enfatiza a relação médico-paciente e integra o melhor das PNCS com o melhor da medicina convencional", englobando aspectos humanísticos, preventivos e curativos dos diversos modelos terapêuticos existentes. A proposta da medicina inlegrativa é criar uma forma em que médicos convencionais e nâo-convencionais possam trabalhar juntos, de forma confortável, em prol da melhora de seus pacientes.

O Program in Integrative Medicine (PIM) ${ }^{64}$ inclui dois anos de bolsas de estudo para quatro médicos residentes, com média de seis anos de experiência clínica prévia. $O$ primeiro ano do currículo é dividido em três módulos didáticos, onđe são ensinados "fundamentos filosóficos", "práticas de estilo de vida" (promoção e prevenção à saúde) e os "diversos sistemas terapêuticos" (fitoterapia, medicina manual, medicina chinesa, homeopatia, medicina energética e alopatia). No segundo ano do treinamento, os residentes passam por quatro módulos de aprimoramento, compostos por "integração clínica" (aplicação do conhecimento teórico na prática clínica), "reflexāo e desenvolvimento pessoal", "educação em pesquisa" e "liderança". Os residentes devem selecionar uma prática médica nåo-convencional para treinamento adicional no segundo ano e são estimulados a experimentar em si próprios as modalidades terapêuticas que recomendam aos seus pacientes. $\mathrm{Na}$ adaptação da medicina integrativa ao currículo da residência médica convencional, cada residente dedica 810 horas semanais ao estudo das PNCS, contabilizando mil horas de instrução no programa de dois anos.

$\mathrm{Na}$ Bruce Rappaport Paculty of Medicine (Haifa, Israel), existe um curso eletivo de introdução às PNCS, dirigido aos wridentes e especialistas do Departamento de Medicina de Família, onde são ensinadas fitoterapia, medicina tradicional chinesa, homeopatia e medicina nutricional, em quatro mó- dulos com 16 sessōes (quatro sessōes/módulo/terapêutica). Esta iniciativa induziu uma mudança positiva na atitude e no interesse dos médicos em relação às PNCS baseadas em evidências, fazendo com que passassem a recomendar PNCS aos seus pacientes, em vez de encaminhá-los a outros médicos prescritores, além de empregar PNCS neles próprios e em seus familiares ${ }^{70}$.

\section{CONCLUSÃO}

Práticas médicas nāo-convencionais, como a homeopatia e a acupuntura, têm se apresentado como uma opçāo de tratamento para as mais diversas enfermidades humanas, sendo procuradas, nos últimos anos, por um contigente cada vez maior da população mundial.

No Brasil, embora reconhecidas como especialidades médicas pelo CFM, a homeopatia e a acupuntura não estāo inseridas no currículo da maioria das escolas médicas, privando a classe médica do conhecimento dos preceitos básicos destas abordagens terapêuticas, que encaram o doente e sua doença de forma distinta da medicina convencional.

Em consequiência deste afastamento do meio acadêmico, com o desconhecimento da maioria dos médicos sobre seus pressupostos fundamentais, a homeopatia e a acupuntura săo alvos de preconceitos e criticas freqüentes, muitas vezes infundados.

No intuito de superar estes entraves, começam a surgir no meio acadêmico propostas de inserção da homeopatia e da acupuntura no currículo da graduação médica, além do desenvolvimento de projetos de pesquisa clíruca e fundamental segundo estas abordagens.

Acreditamos que a homeopatia e a acupuntura devam ser oferecidas, inicialmente, de forma optativa, aos alunos do $3^{\circ}$ ou $4^{\circ}$ anos da graduação das faculdades de medicina brasileiras e, num segundo momento, de forma obrigatória, por serem especialidades médicas reconhecidas. Considerando a diversidade e a complexidade dos novos tópicos, a carga horária para cada disciplina eletiva deveria corresponder a um mínimo de 60 horas-aula, trazendo aos graduandos conhecimento teórico-científico e vivência prática para que se sintam aptos a aconselhar seus futuros pacientes no uso de PNCS, a o contrário da maioria dos cursos das escolas médicas americanas $^{22}$, que dedicam uma carga horária insuficiente (em média duas horas-aula) para cada PNCS.

Como proposta inicial à situação brasileira, o ensino de homeopatia e acupuntura poderia ser incluído nos dois anos básicos dos programas de residência médica (clínica médica, pediatria, ginecologia e obstetrícia, medicina preventiva, medicina de famnia, etc.), com a intenção de transmitir "aspec- 
tos gerais" das referidas especialidades, nos moldes das disciplinas eletivas citadas anteriormente, com carga horária e programa semelhantes.

Para os residentes que desejarem se aprofundar no conhecimento da homeopatia e da acupuntura, após os dois anos básicos, poderia ser implantado um treinamento específico durante um ou dois anos. Nesse caso, o programa de pósgraduação (lato sensu) ou residência médica seguiriam os ditames e a carga horária estipulados pelas associaçōes nacionais de cada especialidade -Associação Médica Homeopática Brasileira (AMHB); Associação Médica Brasileira de Acupuntura (Amba); Sociedade Médica Brasileira de Acupurntura (SMBA).

Em todas as iniciativas que venham a surgir, as evidências cientificas que respaldem estes modelos terapêuticos, tanto na área da pesquisa básica quanto na área da pesquisa clínica, deverāo fazer parte obrigatória da grade programáti$\mathrm{ca}^{71,72}$, por traduzirem para a linguagem acadêmica pressupostos e concepções distintos dos usualmente ensinados, facilitando a compreensão dos alunos.

Traçando o parorama do ensino de PNCS, esperamos estimular a discussão nas escolas médicas brasileiras para a importância de se adequarem às crescentes iniciativas mundiais nesta área, pois o ensino e a pesquisa universitária em PNCS vêm ao encontro das necessidades da sociedade, que se interessa por novas formas de tratamento, mas não conhece os pressupostos científicos que respaldam a utilizaçāo correta e segura das mesmas.

\section{REFERENCIAS BIBLIOGRÁFICAS}

1. National Center for Complementary and Alternative Medicine. National Institute of Health, Betseda, MD, 2003 (http:// nccam.nih.gov/nccam/fcp/faq/index.html\# what-is).

2. Ferreira $\mathrm{ABH}$. Novo Dicionário Aurélio da Língua Portuguesa. Rio de Janeiro: Nova Fronteira; 1986.

3. Moura VL, Warber SL, James SA. CAM providers' messages to conventional medicine: a qualitative study. Am J Med Qual 2002;17(1):10-4.

4. Machado MH et al. Perfil dos médicos no Brasil. Rio de Janeiro: FIOCRUZ/CFM-MS/PNUD; 1996.

5. Eisenberg DM, et al. Unconventiona! medicine in the United States: prevalence, costs and patterns use. N Eng JMed 1993;328:246-52.

6. Eisenberg $D M$, et al. Trends in alternative medicine use in the United States, 1990-1997: results of a follow-up national survey. JAMA 1998;280;1569-75.
7. Fisher $\mathrm{P}$, Ward A. Complementary medicine in Europe. Bt Med J 1994;309:107-11.

8 Burg MA, Hatch RL, Neims AH. Lifetime use of alternative therapy: a study of Florida residents. South Med J 1998, 91(12):1126-31.

9. Burg MA. Women's use of complementary medicine. Costrbining mainstream medicine with alternative practices. ! Fla Med Assoc 1996;83(7):482-8.

10. Furnham A, Forey J. The attitudes, behaviors, and beliets of patients of conventional vs complementary (alternative) medicine. J Clin Psychol 1994;50:458-69.

11. Veeramah EK, Holmes S. Complementary therapy: compltment or threat to modern medicine? J R Soc Health 2000 . 120(1):42-6.

12. Astin JA. Why patients use alternative medicine: resulm of a national survey. JAMA 1998;279:1548-53.

13. Mendicelli VLSL. Homeopatia: percepção e conduta de clientela de postos de saúde de São Paulo. Tese [Doutora. do]. São Paulo: Faculdade de Saúde Pública, Universida. de de Sāo Paulo; 1994.

14. Moreira GN. Homeopatia em Unidade Básica de Saúde (UBS): um espaço disponivel. [Dissertação]. São Paulo: Faculdade de Saúde Pública, Universidade de São Paulo; 1999.

15. Adler SR, Fosket JR. Disclosing complementary and alternative medicine use in the medical encounter. J Fam Pract 1999;48:453-8.

16. Silverstein DD, Spiegel AD. Are physicians aware of the risks of alternative medicine? J Community Health 2001; 26(3):159-74.

17. Dantas F. Desinformaçāo e deformação no ensino médico: a homeopatia no contexto da farmacologia médica. $\mathrm{R} \mathrm{Br}$ Educ Med 1985;9(1):25-9.

18. White AR, Mitchell A, Ernst E. Familiarization witt complementary medicine: report of a new course for primary caxe physicians. J Altern Complement Med 1996;2(2): 307-14.

19. Gianesella EMF. Homeopatia nas escolas médicas: ensino, assistência e pesquisa no Estado de São Paulo. [Dissertação]. São Paulo: Faculdade de Saúde Pública, Universidade de São Paulo; 1998.

20. Straus SE. Complementary and alternative medicine; challenges and opportunities for American medicine. Acad Med 2000;75:572-3.

21. Konefal $\mathrm{J}$. The challenge of educating physicians aboul complementary and alternative medicine. Acad Med 2002 Sep;77(9):847-50. 
22. Murdoch-Eaton D, Crombie H. Complementary and aiternative medicine in the undergraduate curriculum. Med Teach 2002;24(1):100-2.

23. Hui KK, Zylowska L, Hui EK, Yu JL, Li JJ. Introducing integrative East-West medicine to medical students and residents. J Altern Complement Med 2002;8(4):507-15.

24. Park CM. Diversity, the individual, and proof of efficacy: complementary and alternative medicine in medical education. Am J Public Health 2002;92(10):1568-72.

25. Candini F, Weixin H. Moxibustion for correction of breech presentation: a randomized controlled trial. JAMA 1998;280:1580-4.

26. Karris WS, Gowda M, Kolb JW, et al. A randomized, controlled trial of the effects of remote, intercessory prayer on outcomes in patients admitted to the coronary care unit. Arch Intem Med 1999;159:2273-8. Erratum published in Arch Intern Med 2000;160:1878.

27. Dubey NP. Integrated medicine - many approaches, one service. World Health Forum 1997;18(1):56-8.

28. Norred CL, Zamudio S, Palmer SK. Use of complementary and alternative medicines by surgical patientes. I Am Assoc Nurse Anesth 2000;68:13-8.

29. White A, Hayhoe S, Hart A, Ernst E. Adverse eventes following acupunture: prospective survey of 32000 consultations with doctors and physiotherapists. BMJ 2001;323:485-6.

30. Fugh-Berman A. Herb-drug interactions. Lancet 1999;355:134-8.

31. Linzer M, Konrad TR, Douglas J, et al. Management care, time pressure, and physician job satisfaction: results from the physician worklife study. J Gen Intern Med 2000;15:441-50.

32. Haas JS, Cook EF, Puopolo AL, et al. Is the professional satisfaction of general internists associated with patient satisfaction? J Gen Med 2000;15:122-3.

33. Ernst E, Resch KL, Whie A. Complementary medicine: what physicians think of it. Arch Intern Med 1995;155:2405-8.

34. Pirotta MV, Cohen MM, Kotsirilos V, Farish S]. Complementary therapies: have they become accepted in general practice? Med J Aust 2000;172(3):105-9.

35. Corbin Winslow L, Shapiro H. Physicians want education about complementary and alternative medicine to enhance communication with their patients. Arch Intern Med 2002;162(10):1176-81.

36. Sikand A, Laken M. Pediatricians' experience with and attitudes toward complementary/alternative medicine. Arch Pediatr Adolesc Med 1998;152(11):1059-64.
37. Halliday J, Taylor M, Jenikins A, Reilly D. Medical students and complementary medicine. Compl Ther Med 1993;1(4) suppl:32-3.

38. Haltenhof $\mathrm{H}$, Schumm A, Bühler KE. Komplementärme dizin im Urteil von Studierenden der Medizin: Eine Befragung in Vorklinik und Klinik. Forschende Komplementärmedizin 1997;5:284-91.

39. Rampes H, Sharples F, Maragh S, Fisher P. Introducing complementary medicine into the medical curriculum.J $R$ Soc Med 1997;90(1):19-22.

40. Andritzky W. Medical students and alternative medicine - a survey. Gesundheitswesen 1995;57(6):345-8.

41. Duggan K, Verhoef MJ, Hilsden RJ. First-year medical students and complementary and alternative medicines: attitudes, knowledge and experiences. Ann R Coll Physicians Surg Can 1999;32:157-60.

42. Derr S, Shaikh U, Rosen A, Guadagnino P. Medical students' attitudes toward, knowledge of, and experience with complementary nedicine therapies. Acad Med 1998;73:1020.

43. Greiner KA, Murray JL, Kallail KJ. Medical student interest in alternative medicine. J Altem Complement Med 2000; 6(3):231-4.

44. Owen D, Lewith GT. Complementary and alternative medicine (CAM) in the undergraduate medical curriculum: the Southampton experience. Med Educ 2001;35(1):73-7.

45. Rosenbaum ME, Nisly NL, Ferguson K], Kligman EW. Academic physicians and complementary and alternative medicine: an institutional survey. Am I Med Qual 2002; 17(1):3-9.

46. Kemper KJ, Vincent EC, Scardapane JN. Teaching an integrated approach to complementary, alternative, and mainstream therapies for children: a curriculum evaluation. J Altern Complement Med 1999;5(3):261-8.

47. Cohen JJ. Reckoning with alternative medicine. Acad Med 2000;75:571.

48. Dantas F, Ribeiro CT. Atitudes de estudantes de medicina sobre práticas médicas heterodoxas no Brasil. Rev Bras Educ Med 2002;26(2):99-104.

49. Teixeira MZ, Lin CA, Martins MA. Homeopathy and acupuncture teaching at São Paulo University's Medical School: the undergraduates' attitudes and the elemental curriculum. Article in press.

50. Frenkel M, Arye EB. The growing need to teach about complementary and alternative medicine: questions and challenges. Acad Med 2001;76(3):251-4. 
51. British Medical Association. Complementary medicine: new approaches to good practice. Londres, BMA, 1993.

52. Morgan D, Glanville $H$, Mars $S$, Nathanson V. Education and training in complementary and alternative medicine: a postal survey of UK universites, medical schools and faculties of nurse education. Comp Ther Med 1998;6:6470.

53. Barberis L, de Toni B, Schiavone M, Zicca A, Ghio R. Unconventional medicine teaching at the Universities of the European Union. JAltern Complement Med 2001;7:33743.

54. American College of Physicians. Ann Int Med 1998;128: 576-94.

55. Bhattacharya B. MD programs in the United States with complementary and alternative medicine education opportunities: an ongoing listing. J Altern Complement Med 2000;6(1):77-90.

56. Carlston $M$, Stuart MR, Jonas W. Alternative medicine instruction in medical schools and family practice residency programs. Fam Med 1997;24:559-62.

57. Wetzel MS, Eisenberg DM, Kaptchuck TJ. Courses involving complementary and alternative medicine at U.S. medical schools. JAMA 1998;280:784-7.

58. Ruedy J, Kaufman DM, MacLeod H. Alternative and complementary medicine in Canadian medical schools: a survey. Can Med Assoc J 1999;160:816-7.

59. Tsuruoka K, Tsuruoka Y, Kajii E. Complementary medicine education in Japanese medical schools: a survey. Complement Ther Med 2001;9(1):28-33.

60. Brokaw JJ, Tunnicliff G, Raess BU, Saxon DW. The teaching of complementary and alternative medicine in U.S. medical schools: a survey of course directors. Acad Med 2002; 77:876-81.

61. Melchart $D$, Worku F, Linde $K$, Wagner $H$. The university project Munchener Modell for the integration of naturopathy into research and teaching at the Ludwig-Maximilian University in Munich. Complementary Therapies in Med 1994;2:147-53.

62. Chi C. Integrating traditional medicine into modern health care systems: examining the role of Chinese medicine in Taiwan. Soc Sci Med 1994;39(3):307-21.

63. Kim JS, Kim DH, Lee WK, Suh JS, Song KE, Kang BJ, Park EH, Choi JK. Possibility in unification of oriental and western medicine education by combination of educational curricula. Uisahak 1999;8(2):269-77.

64. Maizes V, Schneider C, Bell I, Weil A. Integrative medical education: development and implementation of a comprehensive curriculum at the University of Arizona. Acad Med 2002;77:851 60.

65. Laken MP, Cosovic S. Introducing alternative/complementary healing to allopathic medical students. J Altern Complement Med 1995;1(1):93-8.

66. Hui KK, Zylowska L, Hui EK, Yu JL, Li JJ. Introducing integrative East-West medicine to medical students and residents.J Aitern Complement Med 2002;8(4):507-15.

67. Strauss SE. Complementary and alternative medicine: challenges and opportunities for American medicine. Acad Med 2000;75:572-3.

68. Associação Médica Homeopática Brasileira. Projeto para a Graduação das Faculdades de Medicina - Cadeira eletiva de Homeopatia. AMHB, 2000.

69. Kliger B, Gordon A, Stuart M, Sierpina V. Suggested curriculum guidelines on complementary and alternative medicine: recomendations of the Society of Teachers of Family Medicine Group on Alternative Medicine. Fam Med 2000;32(1):30-3.

70. Ben-Arye E, Frenkel M. Between metaphor and certainty: teaching an introductory course in complementary medicine. Harefuah 2001;140(9):855-9, 893.

71. Marcus DM. How should altemative medicine be taught to medical students and physicians? Acad Med 2001; 76(3):224-9.

72. Sampson $W$. The need for educational reform in teaching about alternative therapies. Acad Med 2001;76(3):248-50.

\section{Endereço para correspondência}

Marcus Zulian Teixeira

R. Dr. Enéas de Carvalho Aguiar, $155-4^{9}$ andar - bloco 6 05403-900 - Sảo Paulo - SP - Brasil

E-mail: marcus@homeozulian.med.br

Chin An Lin

E-mail: calin@uol.com.br

Milton de Arruda Martins

E-mail: mmartins@usp.br 